\title{
Prognostic impact of final kissing balloon technique after crossover stenting for the left main coronary artery: from the AOI-LMCA registry
}

\author{
Koji Nishida ${ }^{1}$ Mamoru Toyofuku ${ }^{2} \cdot$ Takeshi Morimoto $^{3} \cdot$ Masanobu Ohya $^{4} \cdot$ Yasushi Fuku $^{4} \cdot$ Hirooki Higami $^{5}$. \\ Kyohei Yamaji ${ }^{6}$. Hiromi Muranishi ${ }^{6} \cdot$ Yuhei Yamaji $^{6}$. Daisuke Furukawa ${ }^{1} \cdot$ Tomohisa Tada $^{7}$ - Euihong $\mathrm{Ko}^{2}$. \\ Kazushige Kadota ${ }^{4} \cdot K_{\text {Kenji Ando }}$ • Hiroki Sakamoto ${ }^{7} \cdot$ Takashi Tamura $^{2} \cdot$ Kazuya Kawai $^{1} \cdot$ Takeshi Kimura $^{5}$ - The AOI \\ LMCA Stenting Registry Investigators
}

Received: 25 January 2018 / Accepted: 2 April 2018 / Published online: 24 April 2018

(c) The Author(s) 2018

\begin{abstract}
It is still uncertain whether the final kissing balloon technique (FKBT) is mandatory after crossover stenting for the left main coronary artery (LMCA). Assessing Optimal Percutaneous Coronary Intervention for LMCA (AOI-LMCA) registry, a 6-center retrospective registry, enrolled 1809 consecutive patients for LMCA stenting in Japan. In the present analysis, 5 -year clinical outcomes were compared between non-FKBT $(n=160)$ and FKBT $(n=578)$ groups in patients treated with crossover stenting with drug-eluting stents from the LMCA to the left anterior descending artery. Propensity score-matched analysis was also performed in 160 patient pairs. In the entire study population as well as in the propensity-matched population, the cumulative 5-year incidence of the primary outcome measure (target lesion revascularization: TLR) was not significantly different between the FKBT and non-FKBT groups $(10.7$ versus $14.3 \%, P=0.49$, and 11.8 versus $14.3 \%, P=0.53$, respectively). In the sensitivity analysis by the multivariable Cox proportional hazard model, the effect of FKBT relative to non-FKBT for TLR remained insignificant (adjusted HR 0.89, 95\% CI 0.47-1.69, $P=0.72$ ). Regarding the TLR location, there were no significant differences in the cumulative incidences of TLR for LMCA-only, for the main branch, and for the side branch between the 2 groups $(2.2$ versus $1.3 \%, P=0.93,11.8$ versus $9.1 \%, P=0.71$, and 8.2 versus $7.6 \%, P=0.82$, respectively). FKBT after a 1-stent strategy for LMCA crossover stenting did not affect TLR and other clinical outcomes during 5-year follow-up.

Clinical Trial Registration: Assessing Optimal Percutaneous Coronary Intervention for Left Main Coronary Artery Stenting Registry (AOI LMCA Stenting Registry). http://www.umin.ac.jp/ctr/index/htm/. Unique Identifier: UMIN000014706.
\end{abstract}

Keywords Left main coronary artery $\cdot$ Kissing balloon technique $\cdot$ One-stent strategy

Electronic supplementary material The online version of this article (https://doi.org/10.1007/s12928-018-0522-0) contains supplementary material, which is available to authorized users.

Takeshi Kimura

taketaka@kuhp.kyoto-u.ac.jp

1 Department of Cardiology, Chikamori Hospital, Kochi, Japan

2 Department of Cardiology, Japanese Red Cross Society Wakayama Medical Center, Wakayama, Japan

3 Department of Clinical Epidemiology, Hyogo College of Medicine, Nishinomiya, Japan

4 Department of Cardiology, Kurashiki Central Hospital, Kurashiki, Japan
5 Department of Cardiovascular Medicine, Graduate School of Medicine, Kyoto University, 54 Shogoin Kawahara-cho, Sakyo-ku, Kyoto 606-8507, Japan

6 Department of Cardiology, Kokura Memorial Hospital, Kokura, Japan

7 Department of Cardiology, Shizuoka General Hospital, Shizuoka, Japan 


\section{Introduction}

The current international guidelines have recommended coronary artery bypass grafting (CABG) as a class 1 indication in patients with left main coronary artery (LMCA) disease [1-3]. Percutaneous coronary intervention (PCI) has been more and more frequently performed in patients with LMCA disease with a low-moderate SYNTAX (SYNergy between percutaneous coronary intervention with TAXus and cardiac surgery) score as an alternative to CABG, due to improvements of drug-eluting stents (DES) and advancing technique $[4,5]$. A 1-stent strategy is currently considered a standard stenting strategy for LMCA bifurcation lesions, because a 2-stent strategy is associated with higher rates of adverse events such as target lesion revascularization (TLR), and stent thrombosis (ST) [6, 7]. However, the role of final kissing balloon technique (FKBT) after crossover stenting for the main branch is controversial for lesions at any bifurcation [8]. Particularly, the effects of FKBT for LMCA disease have not been adequately assessed in previous reports [9-13]. Therefore, we sought to compare the long-term clinical outcomes between the 2 groups of patients with and without FKBT after crossover DES stenting from LMCA to the left anterior descending artery (LAD), using data from a large multicenter registry in Japan.

\section{Methods}

\section{Study design and patient population}

The AOI-LMCA (Assessing Optimal percutaneous coronary Intervention for Left Main Coronary Artery) stenting registry is a retrospective, multicenter registry that enrolled 1809 consecutive patients who underwent LMCA stenting with bare-metal stents or DES in 6 Japanese hospitals experienced with LMCA stenting between November 2004 and December 2012. The protocol and details of patient enrollment have been described elsewhere [6].

The current study population comprised 738 patients treated with crossover DES stenting as a 1-stent strategy from LMCA to LAD. Five-year clinical outcomes were compared between the non-FKBT group $(N=160)$ and the FKBT group $(N=578)$ after excluding those patients who had ST-elevation myocardial infarction (MI) with cardiogenic shock, or LMCA-left circumflex coronary artery (LCX) crossover stenting (Fig. 1).

Stenting strategies and use of FKBT were left to the discretion of the operators in 5 of 6 participating centers, except for one center in which culotte was the default bifurcation stenting strategy and FKBT after crossover stenting was mandatory. The Medina classification was used to assess each bifurcation lesion, and true bifurcation was defined as Medina classification $(1,1,1),(1,0,1)$, or $(0,1,1)$ [14]. TLR-main branch was defined as TLR from the LMCA to the LAD, and TLR-side branch was defined as TLR involving the LCX ostium only. After the procedure, aspirin $(100 \mathrm{mg} /$ day) and ticlopidine $(200 \mathrm{mg} /$ day $)$ or clopidogrel ( $75 \mathrm{mg} /$ day) were to be prescribed in all patients. The recommended duration of dual antiplatelet therapy (DAPT) was at least 8-12 months after DES implantation. Actual DAPT duration in each patient was determined by each cardiologist based on the institutional protocol. Follow-up data collection was performed by a review of medical records and followup contact with patients and/or their relatives. The study protocol was approved by the institutional review board in each participating center. Written informed consent from
Fig. 1 Study flow chart. AOI assessing optimal percutaneous coronary intervention for left main coronary artery stenting, $C A B G$ coronary artery bypass grafting, $D E S$ drug-eluting stents, FKBT final kissing balloon technique, $L A D$ left anterior descending coronary artery, $L C X$ left circumflex coronary artery, $L M C A$ left main coronary artery, STEMI ST-segment elevation myocardial infarction

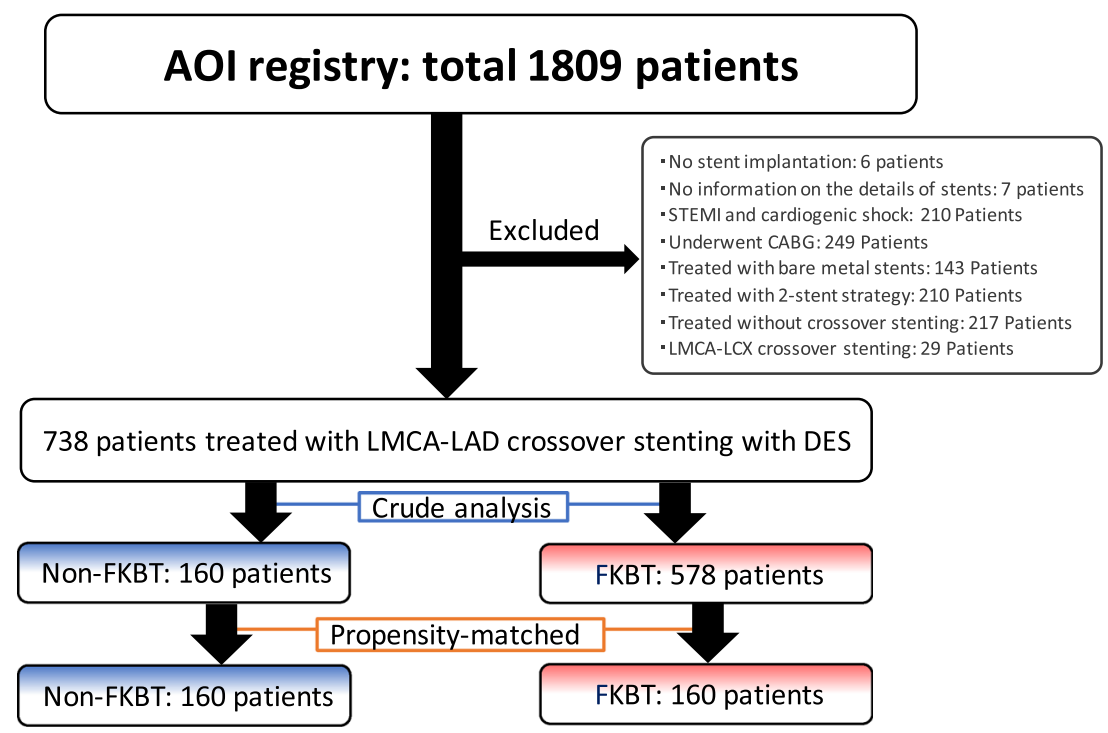


each patient was waived in this retrospective study, because we used clinical information obtained in the routine clinical practice and no patients refused to participate in the study when contacted for follow-up.

\section{Endpoints}

The primary outcome measure in the present analysis was TLR at 5-year. Secondary outcome measures included allcause death, cardiac death, sudden death, MI, definite or probable ST, stroke, any coronary revascularization, or major adverse cardiovascular events (MACE: cardiac death, MI, or TLR) at 5-year. Definitions for each endpoint have been previously described in detail [6].

\section{Statistical analysis}

Categorical variables are presented as counts and percentages, and compared using Chi-squared or Fisher's exact tests. Continuous variables are expressed as mean \pm standard deviation or medians with interquartile range. Continuous variables were compared using Student's $t$ test or the Wilcoxon rank-sum test based on distributions. The cumulative incidence of each endpoint was estimated using the Kaplan-Meier method, and the curves of the 2 groups were compared by the log-rank test.

To adjust the potential confounding for the choice of FKBT, we evaluated the effects of FKBT strategy relative to non-FKBT strategy in a propensity score-matched population. A logistic regression model was used to develop propensity scores for the choice of FKBT strategy with 15 independent variables relevant to the decision regarding FKBT strategy listed in Tables 1 and 2 . The propensity score was then calculated by summing up all coefficient multiples for the corresponding variables (Supplemental Table). To create the propensity score-matched cohort, patients without FKBT were matched to those with FKBT using a 1:1 greedy matching technique [15]. The cumulative incidences of clinical events were compared between FKBT and non-FKBT strategies in the propensity score-matched cohort.

As a sensitivity analysis, the effects of FKBT strategy relative to non-FKBT strategy were evaluated in the entire study population using the multivariable Cox proportional hazard models, and were expressed as hazard ratios (HRs) with $95 \%$ CI. We included 22 clinically relevant factors listed in Tables 1 and 2 as the risk-adjusting variables. Proportional hazard assumptions for the variables were assessed on plots of $\log$ (time) versus $\log$ (log [survival]) stratified by each variable and were verified as acceptable for all variables. As treatment strategies and other related factors changed over time, 3 periods were defined based on the dominant stent types; bare-metal stent period: 2004-2006; first-generation DES (G1-DES) period: 2007-2009; and second-generation DES (G2-DES) period: 2010-2012. The period was used as a stratification variable.

Two physicians (K. Nishida and M. Toyofuku) and a statistician (T. Morimoto) conducted all the statistical analyses using SPSS version 24 (SPSS, Chicago, IL), JMP version 10.0 (SAS Institute, Cary, NC) and SAS version 9.2 (SAS Institute). All reported $P$ values are 2 -sided, and $P$ values $<0.05$ were considered statistically significant.

\section{Results}

\section{Baseline characteristics}

Baseline characteristics were mostly similar between the FKBT and non-FKBT groups in the entire study population, except for the higher prevalence of hemodialysis and peripheral vascular disease in the non-FKBT group. The prevalence of FKBT was significantly different across centers (Table 1). In terms of lesion and procedural characteristics, the prevalence of true bifurcation lesions and mean SYNTAX scores did not differ significantly between the 2 groups (Table 2). Femoral artery was the dominant access site, and G1-DES was implanted in approximately two-thirds of cases without any significant differences between the 2 groups. Patients in the non-FKBT group had significantly higher prevalence of intravascular ultrasound (IVUS) use, as well as lower prevalence of proximal optimization technique, greater number of stents, and larger final balloon size.

In the propensity-matched population of 160 pairs, baseline characteristics were well balanced except for the prevalence of hypertension, peripheral vascular disease, optical coherence tomography (OCT) use, proximal optimization technique, and final balloon size (Tables 1, 2).

\section{Clinical outcomes: FKBT versus non-FKBT}

Median duration of follow-up after the procedure was 3.8 (interquartile range: 2.2-5.3) years. Overall, $83.4 \%$ of patients in this study underwent follow-up coronary artery angiography regardless of the presence of symptoms. In the entire study population, the cumulative 5-year incidence of the primary outcome measure (TLR) was not significantly different between the FKBT and non-FKBT groups (10.7 versus $14.3 \%, P=0.49$ ) (Table 3, and Fig. 2). Regarding the TLR location, there were no significant differences in the cumulative incidences of TLR for LMCA-only, for the main branch, and for the side branch between the 2 groups (2.2 versus $1.3 \%, P=0.93,11.8$ versus $9.1 \%, P=0.71$, and 8.2 versus $7.6 \%, P=0.82$, respectively) (Table 3 ). In the propensity-matched population, the cumulative 5-year incidence of TLR was also not significantly different between the FKBT and non-FKBT groups (11.8 versus $14.3 \%, P=0.53$ ) 
Table 1 Baseline patient characteristics in the entire study population and in the propensity-matched population: FKBT versus non-FKBT

\begin{tabular}{|c|c|c|c|c|c|c|}
\hline & \multicolumn{3}{|c|}{ Entire study population } & \multicolumn{3}{|c|}{ Propensity-matched population } \\
\hline & FKBT $(n=578)$ & Non-FKBT $(n=160)$ & $P$ value & FKBT $(n=160)$ & Non-FKBT $(n=160)$ & $P$ value \\
\hline Age (years) & $72 \pm 10$ & $73 \pm 11$ & 0.34 & $74 \pm 9$ & $73 \pm 11$ & 0.52 \\
\hline Age $\geq 80$ years $^{\mathrm{a}, \mathrm{b}}$ & $141(24 \%)$ & $48(30 \%)$ & 0.15 & $44(28 \%)$ & $48(30 \%)$ & 0.62 \\
\hline Male & $441(76 \%)$ & $117(73 \%)$ & 0.41 & $120(75 \%)$ & $117(73 \%)$ & 0.7 \\
\hline Hypertension & $436(75 \%)$ & $131(82 \%)$ & 0.09 & $115(72 \%)$ & $131(82 \%)$ & 0.03 \\
\hline Diabetes mellitus ${ }^{\mathrm{a}, \mathrm{b}}$ & $258(45 \%)$ & $78(49 \%)$ & 0.36 & $67(42 \%)$ & $78(49 \%)$ & 0.22 \\
\hline Insulin-treated diabetes & $62(11 \%)$ & $23(14 \%)$ & 0.2 & $14(9 \%)$ & $23(14 \%)$ & 0.12 \\
\hline Dyslipidemia & $328(57 \%)$ & $106(66 \%)$ & 0.03 & $91(57 \%)$ & $106(66 \%)$ & 0.09 \\
\hline Current smoker & $80(14 \%)$ & $32(20 \%)$ & 0.06 & $28(18 \%)$ & $32(20 \%)$ & 0.57 \\
\hline $\mathrm{eGFR}\left(\mathrm{mL} / \mathrm{min} / 1.73 \mathrm{~m}^{2}\right)$ & $60.8 \pm 23.1$ & $58.6 \pm 24.3$ & 0.31 & $58.7 \pm 21.4$ & $58.6 \pm 24.3$ & 0.96 \\
\hline Hemodialysis $^{\mathrm{a}, \mathrm{b}}$ & $26(4.5 \%)$ & $14(8.8 \%)$ & 0.036 & $11(6.9 \%)$ & $14(8.8 \%)$ & 0.53 \\
\hline $\begin{array}{l}\text { eGFR }<60 \mathrm{~mL} / \mathrm{min} / 1.73 \mathrm{~m}^{2} \text { and non- } \\
\text { hemodialysis }\end{array}$ & $229(40 \%)$ & $68(43 \%)$ & 0.51 & $66(41 \%)$ & $68(43 \%)$ & 0.82 \\
\hline Previous $\mathrm{PCI}^{\mathrm{a}}$ & $286(50 \%)$ & $75(47 \%)$ & 0.56 & $76(48 \%)$ & $75(47 \%)$ & 0.91 \\
\hline Previous myocardial infarction & $183(32 \%)$ & $46(29 \%)$ & 0.48 & $42(26 \%)$ & $46(29 \%)$ & 0.62 \\
\hline Previous heart failure ${ }^{a, b}$ & $72(13 \%)$ & $18(11 \%)$ & 0.68 & $22(14 \%)$ & $18(11 \%)$ & 0.5 \\
\hline Malignancy $^{\mathrm{a}}$ & $58(10 \%)$ & $19(12 \%)$ & 0.5 & $12(7.5 \%)$ & $19(12 \%)$ & 0.19 \\
\hline Stroke $^{a}$ & $80(14 \%)$ & $22(14 \%)$ & 0.98 & $23(14 \%)$ & $22(14 \%)$ & 0.87 \\
\hline Peripheral vascular disease ${ }^{a}$ & $64(11 \%)$ & $36(23 \%)$ & $<0.0001$ & $21(13 \%)$ & $36(23 \%)$ & 0.03 \\
\hline Euro score & $4.2 \pm 2.4$ & $3.9 \pm 2.5$ & 0.34 & $5.1 \pm 3.0$ & $5.5 \pm 3.5$ & 0.34 \\
\hline Clinical presentation & & & 0.7 & & & 0.57 \\
\hline Stable angina pectoris & $469(81 \%)$ & $132(83 \%)$ & & $128(80 \%)$ & $132(83 \%)$ & \\
\hline UAP/NSTEMI ${ }^{\mathrm{a}, \mathrm{b}}$ & $109(19 \%)$ & $28(18 \%)$ & & $32(20 \%)$ & $28(18 \%)$ & \\
\hline Decompensated heart failure & $38(6.6 \%)$ & $12(7.6 \%)$ & 0.66 & $8(5.0 \%)$ & $12(7.6 \%)$ & 0.34 \\
\hline \multicolumn{7}{|l|}{ Medication } \\
\hline Aspirin & $565(98 \%)$ & $155(98 \%)$ & 0.74 & $157(98 \%)$ & $155(98 \%)$ & 0.7 \\
\hline Thienopyridine & $559(97 \%)$ & $155(98 \%)$ & 0.77 & $157(98 \%)$ & $155(98 \%)$ & 0.7 \\
\hline Warfarin & $42(7.3 \%)$ & $11(6.9 \%)$ & 0.87 & $16(10.0 \%)$ & $11(6.9 \%)$ & 0.32 \\
\hline Statins $^{\mathrm{a}}$ & $410(71 \%)$ & $107(67 \%)$ & 0.32 & $111(69 \%)$ & $107(67 \%)$ & 0.63 \\
\hline$\beta$-Blockers ${ }^{\mathrm{a}}$ & $161(28 \%)$ & $48(30 \%)$ & 0.59 & $43(27 \%)$ & $48(30 \%)$ & 0.54 \\
\hline ACE-I/ARB ${ }^{\mathrm{a}}$ & $337(58 \%)$ & $95(59 \%)$ & 0.81 & $88(55 \%)$ & $95(59 \%)$ & 0.43 \\
\hline Proton pump inhibitors & $272(47 \%)$ & $61(38 \%)$ & 0.047 & $69(43 \%)$ & $61(38 \%)$ & 0.39 \\
\hline $\mathrm{H}_{2}$-blocker & $83(14 \%)$ & $23(15 \%)$ & 0.99 & $22(14 \%)$ & $23(15 \%)$ & 0.85 \\
\hline Time period ${ }^{\mathrm{a}, \mathrm{b}}$ & & & 0.04 & & & 0.01 \\
\hline $\begin{array}{l}\text { Wave 1: 2004-2006 (bare-metal stent } \\
\text { period) }\end{array}$ & $88(15 \%)$ & $37(23 \%)$ & & $37(23 \%)$ & $27(17 \%)$ & \\
\hline Wave 2: 2007-2009 (G1-DES period) & $213(37 \%)$ & $47(29 \%)$ & & $44(28 \%)$ & $69(43 \%)$ & \\
\hline Wave 3: 2010-2012 (G2-DES period) & $277(48 \%)$ & $76(48 \%)$ & & $79(49 \%)$ & $64(40 \%)$ & \\
\hline Institute $^{\mathrm{a}, \mathrm{b}}$ & & & $<0.0001$ & & & 0.9 \\
\hline 1 & $39(6.7 \%)$ & $23(14 \%)$ & & $22(14 \%)$ & $23(14 \%)$ & \\
\hline 2 & $63(11 \%)$ & $53(33 \%)$ & & $50(31 \%)$ & $53(33 \%)$ & \\
\hline 3 & $349(60 \%)$ & $22(14 \%)$ & & $22(14 \%)$ & $22(14 \%)$ & \\
\hline 4 & $94(16 \%)$ & $50(31 \%)$ & & $56(35 \%)$ & $50(31 \%)$ & \\
\hline 5 & $25(4.3 \%)$ & $8(5.0 \%)$ & & $5(3.1 \%)$ & $8(5.0 \%)$ & \\
\hline 6 & $8(1.4 \%)$ & $4(2.5 \%)$ & & $5(3.1 \%)$ & $4(2.5 \%)$ & \\
\hline
\end{tabular}

eGFR estimated glomerular filtration rate, $P C I$ percutaneous coronary intervention, UAP unstable angina pectoris, NSTEMI non-ST-segment elevation myocardial infarction, $A C E-I$ angiotensin-converting enzyme inhibitor, $A R B$ angiotensin II receptor blocker, $G 1$ - $D E S$ first-generation drug-eluting stent, G2-DES second-generation drug-eluting stent

aPotential independent risk-adjusting variables selected for Cox proportional hazards models

${ }^{\mathrm{b}}$ Daggers indicate the variables selected for propensity score matching 
Table 2 Baseline lesion and procedural characteristics in the entire study population and in the propensity-matched population: non-FKBT versus FKBT

\begin{tabular}{|c|c|c|c|c|c|c|}
\hline & \multicolumn{3}{|c|}{ Entire study population } & \multicolumn{3}{|c|}{ Propensity-matched population } \\
\hline & FKBT $(n=578)$ & Non-FKBT $(n=160)$ & $P$ value & $\operatorname{FKBT}(n=160)$ & Non-FKBT $(n=160)$ & $P$ value \\
\hline \multicolumn{7}{|l|}{ Lesion characteristic } \\
\hline CTO in the $\mathrm{RCA}^{\mathrm{a}, \mathrm{b}}$ & $75(13 \%)$ & $20(13 \%)$ & 0.87 & $18(11 \%)$ & $20(13 \%)$ & 0.73 \\
\hline SYNTAX score & $26.0 \pm 9.4$ & $27.2 \pm 9.7$ & 0.13 & $25.6 \pm 8.8$ & $27.3 \pm 9.7$ & 0.1 \\
\hline Bifurcation lesion & $539(93 \%)$ & $146(91 \%)$ & 0.39 & $142(89 \%)$ & $146(91 \%)$ & 0.46 \\
\hline True bifurcation ${ }^{\mathrm{a}, \mathrm{b}}$ & $206(36 \%)$ & $59(37 \%)$ & 0.77 & $62(39 \%)$ & $59(37 \%)$ & 0.73 \\
\hline Extent of coronary artery disease & & & 0.13 & & & \\
\hline Left main only & $43(7 \%)$ & $13(8 \%)$ & & $15(9.4 \%)$ & $13(8.1 \%)$ & \\
\hline Left main +1 vessel & $222(38 \%)$ & $47(29 \%)$ & & $54(34 \%)$ & $47(29 \%)$ & \\
\hline Left main +2 vessels & $212(37 \%)$ & $62(39 \%)$ & & $65(41 \%)$ & $62(39 \%)$ & \\
\hline Left main +3 vessels & $101(18 \%)$ & $38(24 \%)$ & & $26(16 \%)$ & $38(24 \%)$ & \\
\hline Multi-vessel (left main $+\geq 2$ vessels) $)^{a, b}$ & $313(54 \%)$ & $100(63 \%)$ & 0.06 & $91(57 \%)$ & $100(63 \%)$ & 0.31 \\
\hline Medina classification & & & 0.03 & & & 0.28 \\
\hline$(1,0,0)$ & $76(13 \%)$ & $19(12 \%)$ & & $23(14 \%)$ & $19(12 \%)$ & \\
\hline$(0,1,0)$ & $31(5.4 \%)$ & $7(4.4 \%)$ & & $7(4.4 \%)$ & $7(4.4 \%)$ & \\
\hline$(0,0,1)$ & $3(0.5 \%)$ & $6(3.8 \%)$ & & $0(0 \%)$ & $6(3.8 \%)$ & \\
\hline$(1,1,0)$ & $221(38 \%)$ & $52(33 \%)$ & & $50(31 \%)$ & $55(34 \%)$ & \\
\hline$(1,0,1)$ & $21(3.6 \%)$ & $3(1.9 \%)$ & & $6(3.8 \%)$ & $3(1.9 \%)$ & \\
\hline$(0,1,1)$ & $5(0.9 \%)$ & $4(2.5 \%)$ & & $3(1.9 \%)$ & $5(3.1 \%)$ & \\
\hline$(1,1,1)$ & $169(29 \%)$ & $50(31 \%)$ & & $53(33 \%)$ & $51(32 \%)$ & \\
\hline True trifurcation & $71(13 \%)$ & $27(17 \%)$ & 0.15 & $23(15 \%)$ & $27(17 \%)$ & 0.57 \\
\hline Calcified lesion ${ }^{\mathrm{a}, \mathrm{b}}$ & $91(16 \%)$ & $18(11 \%)$ & 0.16 & $18(11 \%)$ & $18(11 \%)$ & 1.0 \\
\hline In-stent restenosis lesion & $15(2.6 \%)$ & $4(2.5 \%)$ & 0.94 & $4(2.5 \%)$ & $4(2.5 \%)$ & 0.99 \\
\hline \multicolumn{7}{|l|}{ Procedural characteristic } \\
\hline Arterial access site & & & 0.04 & & & 0.90 \\
\hline Femoral & $393(68 \%)$ & $122(76 \%)$ & & $123(77 \%)$ & $122(76 \%)$ & \\
\hline Radial or brachial & $185(32 \%)$ & $38(24 \%)$ & & $37(23 \%)$ & $38(24 \%)$ & \\
\hline \multicolumn{7}{|l|}{ Use of mechanical support } \\
\hline IABP & $31(5.4 \%)$ & $10(6.2 \%)$ & 0.67 & $16(10.0 \%)$ & $10(6.3 \%)$ & 0.22 \\
\hline PCPS & $2(0.3 \%)$ & $1(0.6 \%)$ & 0.63 & $1(0.6 \%)$ & $1(0.6 \%)$ & 1.0 \\
\hline Use of rotablator & $42(7.3 \%)$ & $17(11 \%)$ & 0.17 & $11(6.9 \%)$ & $17(11 \%)$ & 0.24 \\
\hline Stent types & & & 0.23 & & & 0.13 \\
\hline G1-DES & $342(59 \%)$ & $103(64 \%)$ & & $90(56 \%)$ & $103(64 \%)$ & \\
\hline G2-DES ${ }^{\mathrm{a}, \mathrm{b}}$ & $236(41 \%)$ & $57(36 \%)$ & & $70(44 \%)$ & $57(36 \%)$ & \\
\hline \multicolumn{7}{|l|}{ Use of intracoronary imaging modalities } \\
\hline IVUS $^{\mathrm{a}, \mathrm{b}}$ & $409(71 \%)$ & $134(84 \%)$ & 0.001 & $127(79 \%)$ & $134(84 \%)$ & 0.31 \\
\hline OCT & $24(4.2 \%)$ & $6(3.8 \%)$ & 0.82 & $15(9.4 \%)$ & $6(3.8 \%)$ & 0.04 \\
\hline None & $145(25 \%)$ & $20(13 \%)$ & 0.001 & $18(11 \%)$ & $20(13 \%)$ & 0.73 \\
\hline Proximal optimization technique & $119(21 \%)$ & $13(8.1 \%)$ & 0.001 & $26(16 \%)$ & $13(8.1 \%)$ & 0.03 \\
\hline Number of stents per lesion & $1.3 \pm 0.5$ & $1.5 \pm 0.7$ & 0.001 & $1.3 \pm 0.6$ & $1.5 \pm 0.7$ & 0.07 \\
\hline Stent size (MV) (mm) & $3.5 \pm 0.7$ & $3.5 \pm 0.6$ & 0.61 & $3.6 \pm 0.6$ & $3.5 \pm 0.6$ & 0.32 \\
\hline Stent size $(\mathrm{MV}) \geq 3.5 \mathrm{~mm}^{\mathrm{a}, \mathrm{b}}$ & $354(61 \%)$ & $104(65 \%)$ & 0.39 & $111(69 \%)$ & $104(65 \%)$ & 0.41 \\
\hline Stent length (MV) (mm) & $26.7 \pm 12.6$ & $26.1 \pm 13.2$ & 0.57 & $26.0 \pm 13.1$ & $26.1 \pm 13.2$ & 0.09 \\
\hline Stent length (MV) $\geq 30 \mathrm{~mm}$ & $141(24 \%)$ & $38(24 \%)$ & 0.87 & $32(20 \%)$ & $38(24 \%)$ & 0.42 \\
\hline Final balloon size (MV) (mm) & $3.5 \pm 0.6$ & $3.7 \pm 0.6$ & 0.003 & $3.5 \pm 0.6$ & $3.7 \pm 0.6$ & 0.004 \\
\hline Maximum balloon size (SV) (mm) & $2.5 \pm 0.6$ & - & - & $2.4 \pm 0.5$ & - & - \\
\hline
\end{tabular}

CTO chronic total occlusion, $R C A$ right coronary artery, SYNTAX SYNergy between PCI with TAXus and Cardiac Surgery, IABP intra-aortic balloon pumping, PCPS percutaneous cardiopulmonary support, IVUS intravascular ultrasound, $O C T$ optical coherence tomography, $M V$ main vessel, $S V$ side vessel. Other abbreviations are the same as in Table 1

${ }^{a}$ Potential independent risk-adjusting variables selected for Cox proportional hazards models

${ }^{\mathrm{b}}$ Daggers indicate independent model for variables selected for propensity score matching 
Table 3 Five-year clinical outcomes in the entire study population and in the propensity-matched population: non-FKBT and FKBT

\begin{tabular}{|c|c|c|c|c|c|c|}
\hline & \multicolumn{3}{|c|}{ Entire study population } & \multicolumn{3}{|c|}{ Propensity-matched population } \\
\hline & \multicolumn{3}{|c|}{$\begin{array}{l}\text { Patients with at least } 1 \text { event (cumulative } 5 \text {-year } \\
\text { incidence, } \% \text { ) }\end{array}$} & \multicolumn{3}{|c|}{$\begin{array}{l}\text { Patients with at least } 1 \text { event (cumulative 5-year } \\
\text { incidence, \%) }\end{array}$} \\
\hline & FKBT $(n=578)$ & Non-FKBT $(n=160)$ & $P$ value & FKBT $(n=160)$ & Non-FKBT $(n=160)$ & $P$ value \\
\hline TLR & $59(10.7)$ & $17(14.3)$ & 0.49 & $17(11.8)$ & $17(14.3)$ & 0.53 \\
\hline TLR-LMCA only & $7(1.3)$ & $2(2.2)$ & 0.93 & $1(0.6)$ & $2(2.2)$ & 0.56 \\
\hline TLR-main branch & $54(9.1)$ & $15(11.8)$ & 0.71 & $14(10.4)$ & $15(12.5)$ & 0.5 \\
\hline TLR-side branch & $41(7.6)$ & $11(8.2)$ & 0.82 & $10(6.7)$ & $11(8.2)$ & 0.59 \\
\hline All-cause death & $98(19.9)$ & $36(23.1)$ & 0.23 & $29(21.2)$ & $36(23.1)$ & 0.6 \\
\hline Cardiac death & $30(6.3)$ & $18(9.1)$ & 0.14 & $11(8.1)$ & $18(9.1)$ & 0.68 \\
\hline Sudden death & $9(1.9)$ & $5(2.0)$ & 0.61 & $2(1.5)$ & $5(2.0)$ & 0.64 \\
\hline Myocardial infarction & $12(2.6)$ & $8(6.4)$ & 0.06 & $6(4.1)$ & $8(6.6)$ & 0.57 \\
\hline Definite or probable stent thrombosis & $2(0.3)$ & $1(0.6)$ & 0.62 & $2(1.3)$ & $1(0.6)$ & 0.57 \\
\hline Stroke & $25(5.0)$ & $6(4.5)$ & 0.87 & $7(4.2)$ & $6(4.5)$ & 0.75 \\
\hline Ischemic & $21(4.2)$ & $3(2.2)$ & 0.51 & $5(2.8)$ & $3(2.2)$ & 0.99 \\
\hline Hemorrhagic & $4(0.8)$ & $3(2.2)$ & 0.16 & $2(1.4)$ & $3(2.2)$ & 0.64 \\
\hline Any coronary revascularization & $160(29.9)$ & $42(34.1)$ & 0.75 & $39(26.4)$ & $42(34.1)$ & 0.24 \\
\hline MACE & $92(17.0)$ & $34(21.3)$ & 0.24 & $27(18.7)$ & $34(21.3)$ & 0.45 \\
\hline
\end{tabular}

The number of patients with at least 1 event was counted through the entire follow-up period, while the cumulative incidence was truncated at 5 years

$P$ values estimated by the log-rank test

$M A C E$ major adverse cardiac events, TLR target lesion revascularization

(Table 3, and Fig. 2). In the sensitivity analysis by the multivariable Cox proportional hazard model, the effect of FKBT relative to non-FKBT for TLR remained insignificant (adjusted HR 0.89, 95\% CI 0.47-1.69, $P=0.72$ ) (Table 4). Cumulative 5-year incidences of the secondary outcome measures including MACE were also not significantly differences between the FKBT and non-FKBT groups both in the entire study population and in the propensity-matched population (Table 3 and Fig. 3). The effects of FKBT relative to non-FKBT for the secondary outcome measures were also not significant (Table 4).

\section{Discussion}

The main findings of the present study from a large multicenter registry in Japan were the followings; (1) FKBT after a 1-stent strategy for LMCA crossover stenting did not affect TLR and other clinical outcomes during 5-year follow-up; (2) FKBT also did not affect the location of TLR (Fig. 3).

FKBT has been widely performed at any bifurcation on the expectations of optimizing stent apposition at the main branch, ameliorating the side branch ostial narrowing caused by carina shift, reducing strut protrusion into the side branch ostium, and maintaining access to the side branch [16]. In contrast, FKBT might be associated with injury of the side branch ostium potentially leading to restenosis, stent deformation related to over-dilation of the stent proximal to the side branch, and strut mal-apposition due to inadequate rewiring position $[17,18]$. FKBT is generally advocated after a two-stent strategy for any bifurcation [19, 20]. However, the clinical benefits of routine FKBT after a 1-stent strategy appear uncertain at any bifurcation lesion according to the several previous studies [8-11]. The role of FKBT at LMCA bifurcation might be markedly different from that at any other bifurcation, because LMCA bifurcation involves LCX, which is often a large vessel and supplies a large myocardial territory. FKBT would be justified in cases of hemodynamically significant ostial LCX stenosis or flow impairment of LCX after crossover stenting. However, it remains uncertain whether FKBT should be performed in cases of preserved LCX flow or without significant stenosis at ostial LCX ostium after LMCA crossover stenting.

Crossover stenting for the LMCA as a 1-stent strategy is forced to jail the LCX ostium by stent struts. There were concerns on the possibility that formation of neointima and thrombus at the jailed strut with compromise of LCX flow may increase the risk of ST or TLR [21]. A OCT study noted that FKBT may reduce the frequency of uncovered struts and subclinical thrombus at the side branch orifice, although FKBT did not decrease MACE and TLR in this small cohort [22]. The COBIS II registry, which included LMCA lesions in $26 \%$ of cases, demonstrated that a 1 -stent technique with FKBT for any bifurcation lesions was associated with 


\section{TLR}

\section{A: Crude}

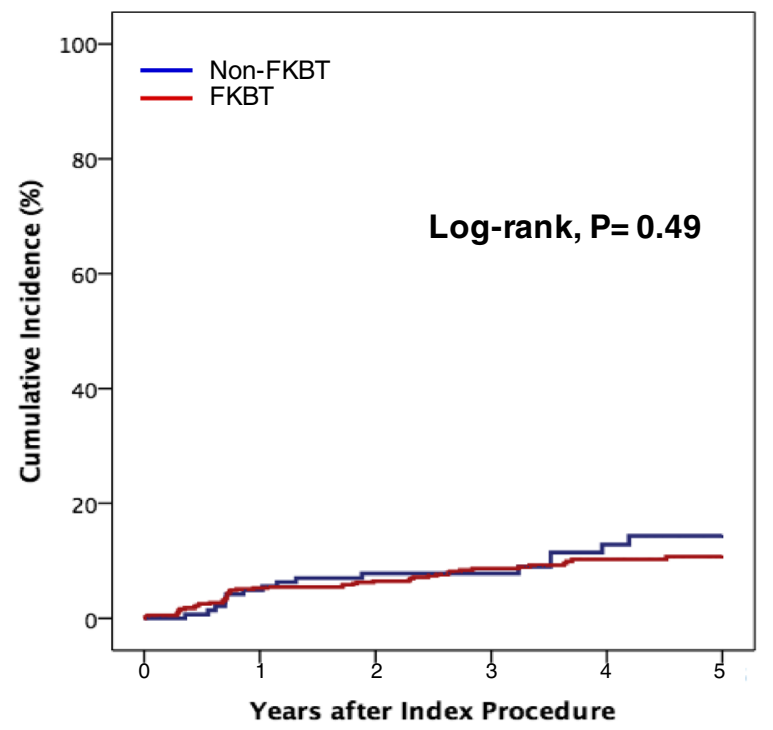

\begin{tabular}{|lccccc|}
\multicolumn{1}{c}{$\mathbf{0}$} & $\mathbf{3 0}$ days & $\mathbf{1}$ year & $\mathbf{3}$ years & $\mathbf{5}$ years \\
\hline Non-FKBT & & & & & \\
\hline Cumulative incidence & & $0.0 \%$ & $4.8 \%$ & $7.8 \%$ & $14.3 \%$ \\
Number of patients with event & & 0 & 7 & 12 & 16 \\
Number of patients at risk & 160 & 155 & 136 & 78 & 41 \\
\hline FKBT & & & & & \\
\hline Cumulative incidence & $0.7 \%$ & $5.2 \%$ & $8.6 \%$ & $10.7 \%$ \\
Number of patients with event & & 3 & 29 & 44 & 50 \\
Number of patients at risk & 578 & 567 & 509 & 336 & 151 \\
\hline
\end{tabular}

B: Propensity-matched

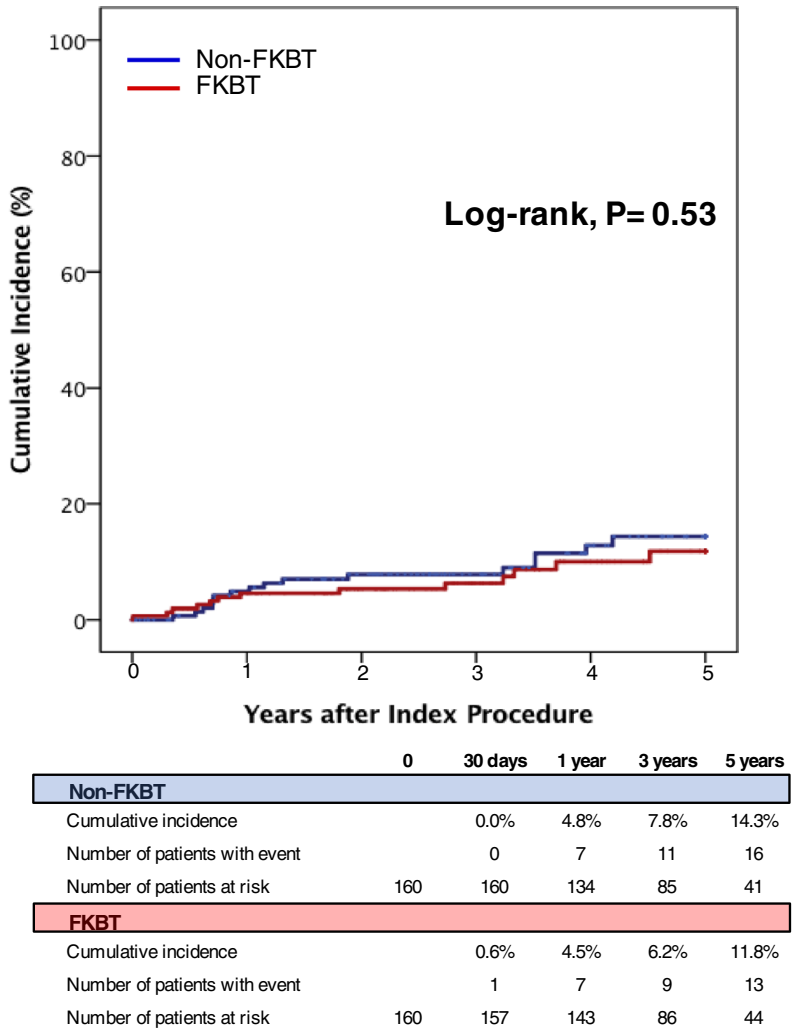

Fig. 2 Kaplan-Meier curves for TLR in the entire study population and in the propensity-matched population: FKBT versus Non-FKBT. FKBT final kissing balloon technique, TLR target lesion revascularization

Table 4 Effects of FKBT relative to non-FKBT for clinical outcomes in the crude population

\begin{tabular}{|c|c|c|c|c|}
\hline & Unadjusted HR (95\% CI) & $P$ value & Adjusted HR (95\% CI) & $P$ value \\
\hline TLR & $0.82(0.47-1.4)$ & 0.49 & $0.89(0.47-1.69)$ & 0.72 \\
\hline All-cause death & $0.78(0.51-1.18)$ & 0.24 & $0.69(0.43-1.12)$ & 0.14 \\
\hline Cardiac death & $0.6(0.31-1.19)$ & 0.15 & $0.41(0.18-0.94)$ & 0.03 \\
\hline Sudden death & $0.71(0.19-2.67)$ & 0.61 & $0.31(0.06-1.56)$ & 0.15 \\
\hline Myocardial infarction & $0.42(0.16-1.01)$ & 0.07 & $0.55(0.17-1.79)$ & 0.32 \\
\hline Definite or probable stent thrombosis & N/A & N/A & N/A & N/A \\
\hline Stroke & $0.93(0.37-2.3)$ & 0.87 & $0.89(0.32-2.46)$ & 0.82 \\
\hline Ischemic & $1.5(0.44-5.12)$ & 0.52 & $1.15(0.29-4.5)$ & 0.84 \\
\hline Hemorrhagic & $0.36(0.08-1.59)$ & 0.18 & $0.71(0.12-4.29)$ & 0.71 \\
\hline Any coronary revascularization & $0.95(0.67-1.34)$ & 0.75 & $0.81(0.55-1.2)$ & 0.3 \\
\hline MACE & $0.78(0.51-1.19)$ & 0.25 & $0.64(0.39-1.06)$ & 0.08 \\
\hline
\end{tabular}

Effect of FKBT relative to non-FKBT is expressed as a hazard ratio with the $95 \%$ confidence interval by Cox proportional hazard models $C I$ confidence interval, $H R$ hazard ratio, N/A not assessed. Other abbreviations are the same as in Table 3

favorable long-term clinical outcomes, whereas a 1-stent strategy with FKBT for LMCA was not associated with better MACE outcomes compared with a 1-stent strategy without FKBT [11]. Furthermore, two recent single center registries found that midterm clinical outcomes were not significantly different regardless of FKBT after crossover stenting of the LMCA $[12,13]$. As in the previous reports, the present multicenter study with longer follow-up, with a 


\section{MACE}

\section{A: Crude}

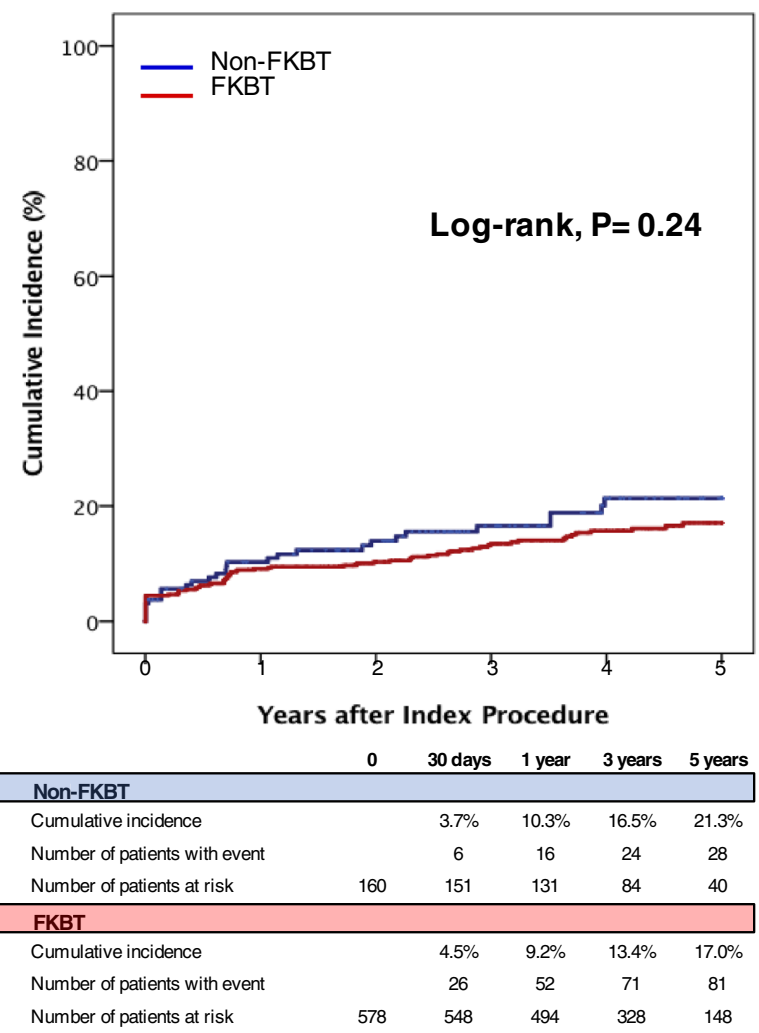

B: Propensity-matched

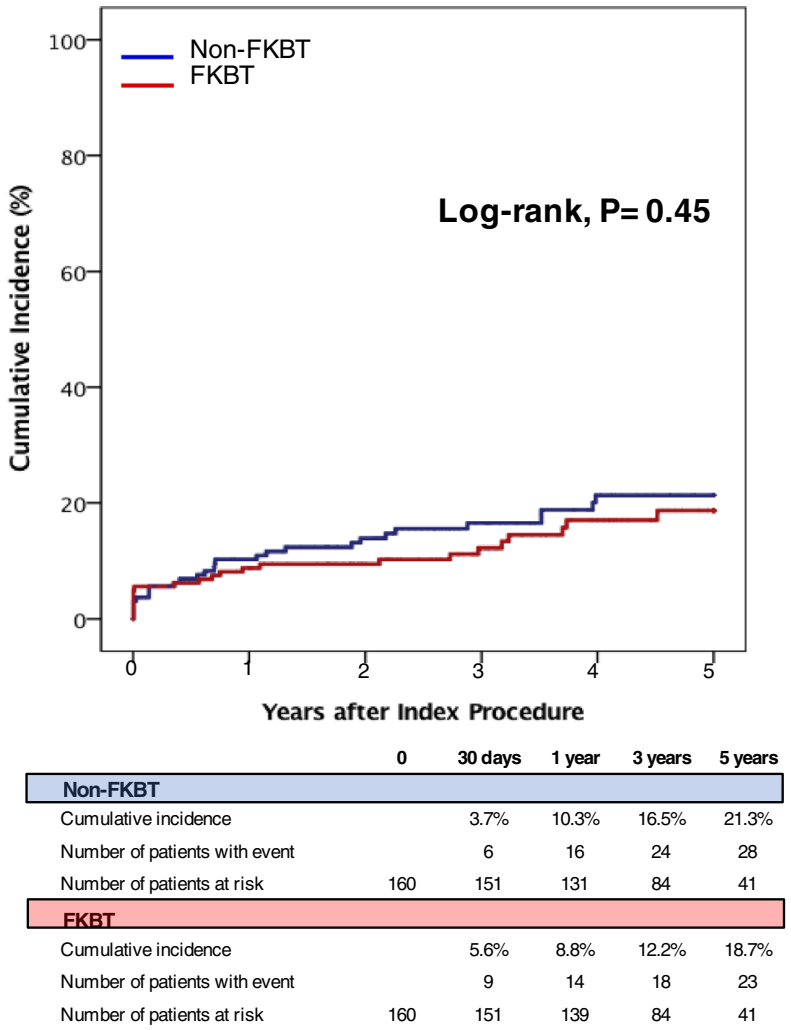

Fig. 3 Kaplan-Meier curves for MACE in the entire study population and in the propensity-matched population: FKBT versus Non-FKBT. FKBT final kissing balloon technique, MACE major adverse cardiac events

higher frequency of FKBT, and with TLR as the primary outcome measure did not demonstrate superiority of FKBT strategy over non-FKBT strategy.

Interestingly, the frequency of FKBT after crossover stenting for the LMCA in the present study was higher (78\%) than those reported in previous studies (32-35\%) [11, 12]. Reflecting the operator preferences, there is a wide variation across countries and institutions, regarding the performance of FKBT after crossover stenting for LMCA. The reason for the high frequency of FKBT in our registry might be that FKBT has been mainly performed not only for decreased coronary blood flow, but also for opening the jailed strut with FKBT to maintain access to the LCX for future PCI. The rationale for this "prophylactic" FKBT would be a concern about stent deformation and restenosis caused by balloon dilatation for a stable jailed strut of the LCX ostium and for stable LMCA in cases that need TVR for LCX in the future.

Despite concerns of increased TLR rate for the proximal LMCA regarding polymer damage, strut deformation, and asymmetry of the proximal stent by the hugging balloon, which potentially decrease tissue concentrations of the eluted drug, TLR rate for the proximal LMCA did not differ significantly regardless of FKBT in the present study [23]. The frequency of TLR for LCX involving the ostium was similar with or without FKBT in the present study, despite concerns about balloon injury or incomplete stent apposition with FKBT. Therefore, FKBT would not be harmful in terms of midterm outcomes in this study. One of the reasons for these neutral results might be that FKBT was not harmful despite its potential risks, because the frequency of IVUS use was higher than in previous reports [9-12]. Guidance with IVUS or OCT is useful particularly for the LMCA for confirming side branch distal rewiring before FKBT and determination of stent and balloon sizes $[18,24]$. However, FKBT often needs a higher volume of contrast, longer procedure time, and more devices than no FKBT $[9,12]$. Based on our present results, FKBT may not be mandatory in cases without flow limitation after stenting. Further and longer term validation study would be required for this issue. 


\section{Limitations}

The present study has several important limitations. First, this registry was conducted as a nonrandomized and retrospective observational study. Therefore, we performed propensity-matched analysis to adjust for the potential confounders. Nevertheless, we could not deny the presence of unmeasured confounders and selection bias. Second, we did not assess the anatomic assessments such as the extent of myocardial territory and of previous myocardial infarction or diameter of LCX, and the degree of stenosis at the LCX ostium after crossover stenting, which might be closely related to the decision whether to perform FKBT. Particularly, significance of LCX territory or viability may affect the clinical outcome. Additionally, patients treated with 2-stent strategy for bailout subsequent to FKBT was excluded in this study cohort, who might have been benefitted from LCX intervention. Third, physiological assessment such as fractional flow reserve was not adequately analyzed, although angiography often overestimate the stenosis of the LCX ostium compared with functional assessment [25]. Fourth, the frequency of follow-up coronary artery angiography was high compared to previous reports, which might have increased the incidence of angiography-driven TLR. Fifth, the sample size was not large enough to evaluate those clinical outcomes such as ST, MI, and cardiac death. Indeed, the risk for MI and cardiac death favored FKBT, although we could not draw definitive conclusions. Finally, G1-DES were used in a large proportion of patients. Use of newer generation DES has been shown to improve clinical outcomes after LMCA stenting $[4,5]$.

\section{Conclusion}

FKBT after a 1-stent strategy for LMCA crossover stenting did not affect TLR and other clinical outcomes during 5-year follow-up.

Acknowledgements The authors would like to thank the members of the catheterization laboratories and physicians of the participating centers.

\section{Compliance with ethical standards}

Conflict of interest Takeshi Kimura serves as an advisory board member for Abbott Vascular. The other authors declare that they have no conflict of interest.

Open Access This article is distributed under the terms of the Creative Commons Attribution 4.0 International License (http://creativeco mmons.org/licenses/by/4.0/), which permits unrestricted use, distribution, and reproduction in any medium, provided you give appropriate credit to the original author(s) and the source, provide a link to the Creative Commons license, and indicate if changes were made.

\section{References}

1. Fihn SD, Gardin JM, Abrams J, Berra K, Blankenship JC, Dallas AP, et al. 2012 ACCF/AHA/ACP/AATS/PCNA/ SCAI/STS Guideline for the diagnosis and management of patients with stable ischemic heart disease. J Am Coll Cardiol. 2012;60:e44-164.

2. Fihn SD, Blankenship JC, Alexander KP, Bittl JA, Byrne JG, Fletcher BJ, et al. 2014 ACC/AHA/AATS/PCNA/SCAI/STS focused update of the guideline for the diagnosis and management of patients with stable ischemic heart disease. J Am Coll Cardiol. 2014;64:1929-49.

3. Windecker S, Kolh P, Alfonso F, Collet JP, Cremer J, Falk V, et al. 2014 ESC/EACTS guidelines on myocardial revascularization. Eur Heart J. 2014;35:2541-619.

4. Stone GW, Sabik JF, Serruys PW, Simonton CA, Généreux P, Puskas J, et al. Everolimus-eluting stents or bypass surgery for left main coronary artery disease. N Engl J Med. 2016;375:2223-35.

5. Mäkikallio T, Holm NR, Lindsay M, Spence MS, Erglis A, Menown IB, et al. Percutaneous coronary angioplasty versus coronary artery bypass grafting in treatment of unprotected left main stenosis (NOBLE): a prospective, randomised, open-label, non-inferiority trial. Lancet. 2016;388:2743-52.

6. Ohya M, Kadota K, Toyofuku M, Morimoto T, Higami H, Fuku $\mathrm{Y}$, et al. Long-term outcomes after stent implantation for left main coronary artery (from the Multicenter Assessing Optimal Percutaneous Coronary Intervention for Left Main Coronary Artery Stenting Registry). Am J Cardiol. 2017;119:355-64.

7. Takagi K, Naganuma T, Chieffo A, Fujino Y, Latib A, Tahara S, et al. Comparison between 1- and 2-stent strategies in unprotected distal left main disease: the Milan and New-Tokyo Registry. Circ Cardiovasc Interv. 2016;9:e003359.

8. Liu G, Ke X, Huang ZB, Wang LC, Huang ZN, Guo Y, et al. Final kissing balloon inflation for coronary bifurcation lesions treated with single-stent technique: a meta-analysis. Herz. 2017. https:// doi.org/10.1007/s00059-017-4647-1.

9. Niemelä M, Kervinen K, Erglis A, Holm NR, Maeng M, Christiansen EH, et al. Randomized comparison of final kissing balloon dilatation versus no final kissing balloon dilatation in patients with coronary bifurcation lesions treated with main vessel stenting: the Nordic-Baltic Bifurcation Study III. Circulation. 2011;123:79-86.

10. Gwon HC, Hahn JY, Koo BK, Song YB, Choi SH, Choi JH, et al. Final kissing ballooning and long-term clinical outcomes in coronary bifurcation lesions treated with 1-stent technique: results from the COBIS registry. Heart. 2012;98:225-31.

11. Yu CW, Yang JH, Song YB, Hahn JY, Choi SH, Choi JH, et al. Long-term clinical outcomes of final kissing ballooning in coronary bifurcation lesions treated with the 1-stent technique: results from the COBIS II Registry (Korean Coronary Bifurcation Stenting Registry). JACC Cardiovasc Interv. 2015;8:1297-307.

12. Gao Z, Xu B, Yang YJ, Qiao SB, Wu YJ, Chen T, et al. Effect of final kissing balloon dilatation after one-stent technique at left-main bifurcation: a single center data. Chin Med J. 2015;128:733-9.

13. Ahn JM, Lee PH, Park DW, Kang SJ, Lee SW, Kim YH, et al. Benefit of final kissing balloon inflation mandatory after simple crossover stenting for left main bifurcation narrowing. Am J Cardiol. 2017;119:528-34.

14. Lassen JF, Holm NR, Banning A, Burzotta F, Lefèvre T, Chieffo A, et al. Percutaneous coronary intervention for coronary 
bifurcation disease: 11th consensus document from the European Bifurcation Club. EuroIntervention. 2016;12:38-46.

15. Austin PC. Balance diagnostics for comparing the distribution of baseline covariates between treatment groups in propensity score matched samples. Stat Med. 2009;28:3083-107.

16. Sgueglia GA, Chevalier B. Kissing balloon inflation in percutaneous coronary interventions. JACC Cardiovasc Interv. 2012;5:803-11.

17. Rahman S, Leesar T, Cilingiroglu M, Effat M, Arif I, Helmy T, et al. Impact of kissing balloon inflation on the main vessel stent volume, area, and symmetry after side-branch dilation in patients with coronary bifurcation lesions: a serial volumetric intravascular ultrasound study. JACC Cardiovasc Interv. 2013;6:923-31.

18. Okamura T, Nagoshi R, Fujimura T, Murasato Y, Yamawaki $\mathrm{M}$, Ono S, et al. Impact of guidewire recrossing point into stent jailed side branch for optimal kissing balloon dilatation: core lab 3D optical coherence tomography analysis. EuroIntervention. 2018;13:e1785-93.

19. Hoye A, Iakovou I, Ge L, van Mieghem CA, Ong AT, Cosgrave $\mathrm{J}$, et al. Long-term outcomes after stenting of bifurcation lesions with the "crush" technique: predictors of an adverse outcome. J Am Coll Cardiol. 2006;47:1949-58.

20. Adriaenssens T, Byrne RA, Dibra A, Iijima R, Mehilli J, Bruskina $\mathrm{O}$, et al. Culotte stenting technique in coronary bifurcation disease: angiographic follow-up using dedicated quantitative coronary angiographic analysis and 12-month clinical outcomes. Eur Heart J. 2008;29:2868-76.

21. Nakazawa G, Yazdani SK, Finn AV, Vorpahl M, Kolodgie FD, Virmani R. Pathological findings at bifurcation lesions: the impact of flow distribution on atherosclerosis and arterial healing after stent implantation. J Am Coll Cardiol. 2010;55:1679-87.

22. Hariki H, Shinke T, Otake H, Shite J, Nakagawa M, Inoue T, et al. Potential benefit of final kissing balloon inflation after single stenting for the treatment of bifurcation lesions-insights from optical coherence tomography observations. Circ J. 2013;77:1193-201.

23. Kim YH, Lee JH, Roh JH, Ahn JM, Yoon SH, Park DW, et al. Randomized comparisons between different stenting approaches for bifurcation coronary lesions with or without side branch stenosis. JACC Cardiovasc Interv. 2015;8:550-60.

24. Andell P, Karlsson S, Mohammad MA, Götberg M, James S, Jensen $\mathbf{J}$, et al. Intravascular ultrasound guidance is associated with better outcome in patients undergoing unprotected left main coronary artery stenting compared with angiography guidance alone. Circ Cardiovasc Interv. 2017;10:e004813.

25. Koo BK, Waseda K, Kang HJ, Kim HS, Nam CW, Hur SH, et al. Anatomic and functional evaluation of bifurcation lesions undergoing percutaneous coronary intervention. Circ Cardiovasc Interv. 2010;3:113-9. 